MITSUBISHI ELECTRIC RESEARCH LABORATORIES

http://www.merl.com

\title{
Sub-optimal Control Design of a Semi-active Vibration Reduction System
}

\author{
Wang, Y.; Utsunomiya, K.
}

TR2013-038 June 2013

\begin{abstract}
This paper considers the vibration reduction of transportation systems using semi-active actuators to improve the ride quality. Control design for the resultant semi-active system is difficult for nonlinear dynamics, constrained control, lack of performance-oriented nonlinear control design, and limited state information. A sub-optimal control structure is proposed to address the performance requirement by mimicking the optimal control. A specific sub-optimal control is provided and implemented with one measurement to reduce the hardware cost. Performance analysis of the sub-optimal control is investigated. The semi-active system with the suboptimal control is simulated to demonstrate the effectiveness.
\end{abstract}

American Control Conference (ACC)

This work may not be copied or reproduced in whole or in part for any commercial purpose. Permission to copy in whole or in part without payment of fee is granted for nonprofit educational and research purposes provided that all such whole or partial copies include the following: a notice that such copying is by permission of Mitsubishi Electric Research Laboratories, Inc.; an acknowledgment of the authors and individual contributions to the work; and all applicable portions of the copyright notice. Copying, reproduction, or republishing for any other purpose shall require a license with payment of fee to Mitsubishi Electric Research Laboratories, Inc. All rights reserved.

Copyright (C) Mitsubishi Electric Research Laboratories, Inc., 2013

201 Broadway, Cambridge, Massachusetts 02139 



\title{
Sub-optimal control design of a semi-active vibration reduction system
}

\author{
Yebin Wang and Kenji Utsunomiya
}

\begin{abstract}
This paper considers the vibration reduction of transportation systems using semi-active actuators to improve the ride quality. Control design for the resultant semi-active system is difficult for nonlinear dynamics, constrained control, lack of performance-oriented nonlinear control design, and limited state information. A sub-optimal control structure is proposed to address the performance requirement by mimicking the optimal control. A specific sub-optimal control is provided and implemented with one measurement to reduce the hardware cost. Performance analysis of the sub-optimal control is investigated. The semi-active system with the suboptimal control is simulated to demonstrate the effectiveness.
\end{abstract}

\section{INTRODUCTION}

Vibration reduction of transportation systems is to meet the requirement for ride comfort. Existing architectures for the vibration reduction of transportation systems fall into three categories: passive, fully active, and semi-active, where passive components, active actuators, or semi-active actuators are used in the respective architecture. A passive system is reliable and low-cost but with limited performance. The active architecture, comprising of control mechanism and fully active actuators, leads to superior performance at the expense of a high first cost, relatively large electric power requirements and potentially reduced reliability [1]. The semi-active architecture was originally proposed in [2] to trade off the performance of vibration reduction and the system cost. The semi-active architecture takes a similar form of the active counterpart except that the fully active actuators are substituted by semi-active actuators. A wide range of study on semi-active systems, mainly on automotive suspensions, demonstrates that a semi-active system can achieve comparable performance of its active counterpart at a reduced first cost and potentially simplify power supply requirements [1].

The application of semi-active actuators such as MR dampers renders a challenging problem-control design of the semi-active system subject to performance criteria. The dissipative constraint on semi-active dampers not only introduces nonlinearity, but also leads to constrained control. Work, e.g. [2], [3], [4], first performs control design for a fully active system, then derives semi-active control laws by 'clipping' active control laws to ensure that semi-active actuators generate forces in the same directions as active actuators would. The aforementioned two-step design approach

Yebin Wang is with Mitsubishi Electric Research Laboratories, Cambridge, MA, 02139 USA. (email: yebinwang@merl.com)

Kenji Utsunomiya is with Advanced Technology R\&D Center, Mitsubishi Electric Corporation, 8-1-1, Tsukaguchi-honmachi, Amagasaki City, 6618661, Japan. (email: Utsunomiya.Kenji@ db.MitsubishiElectric.co.jp) is straightforward since the fully active system is linear time invariant (LTI). Commonly used active control strategies include Sky-Hook [2], Ground-Hook [5], LQR/LQG [6], and $\mathcal{H}_{\infty}$ [7]. This approach however does not address the nonlinear dynamics during the stage of control synthesis. Work [8], [9] represents some of numerous efforts to establish the control of a semi-active automotive suspension by treating it as a bilinear system. Rigorous derivation shows how the dissipative and saturation constraints lead to the performance loss of a semi-active system from its active counterpart. The optimal control requires the solution of switching differential Riccati equations and is not in the form of state feedback. Nonlinear design such as the Lyapunov-based control [10], decentralized bang-bang control [11], establishes the semiactive control laws by maximizing the dissipative rate of distinctive energy functions. One of the disadvantages of these approaches is that the performance of the closed-loop system is not guaranteed for the lack of connection between performance costs and energy functions. Representing the semiactive system as a linear hybrid system, work [12] considers a sub-optimal control using hybrid model predictive control approach. Recent work [13] performs nonlinear $\mathcal{H}_{\infty}$ control design of semi-active automotive suspensions. By restricting the nonlinear constrained weights on controlled signals, the Hamilton-Jacobi inequality is condensed into an algebraic Riccati inequality [14]. Although a smooth nonlinear control is guaranteed, this approach does not ensures the dissipative condition in design stage. Readers are referred to [15] for a more detailed survey of semi-active controls.

This paper considers the vibration reduction of a simplified quarter car model using semi-active dampers with adjustable viscous damping coefficients. In Section II we introduce the semi-active system, and state the problem to be solved. In Section III we discuss the optimal control of a semi-active system, propose a general sub-optimal control structure, and implement a specific sub-optimal control with one measurement. Performance analysis of the sub-optimal control is investigated in Section IV. Simulation results of several semiactive control laws are provided in Section IV to demonstrate the effectiveness of the proposed sub-optimal control and the tuning methodology. Section $\mathrm{V}$ concludes this note.

Notation: $\|x(t)\|_{p}$ is the $\mathcal{L}_{p}$-norm of $x(t)$, for $1 \leq p<$ $\infty$. A positive (or negative) definite (p.d.f) matrix $P$ (or $Q$ ) is abbreviated by $P>0$ (or $Q<0$ ).

\section{PRELIMINARY}

\section{A. The Semi-Active System}

We consider a quarter car model which is a two degree of freedom (2DOF) system as shown in Figure 1. The 2DOF 
system consists of a first mass $\left(m_{1}\right)$, a second mass $\left(m_{2}\right)$, a road profile, a controller $(C)$, sensors $(S)$, dampers $\left(b_{1}, b_{2}\right)$ and springs $\left(k_{1}, k_{2}\right)$. The semi-active actuator $\left(b_{2}\right)$ is placed between the second mass and the road profile. The system

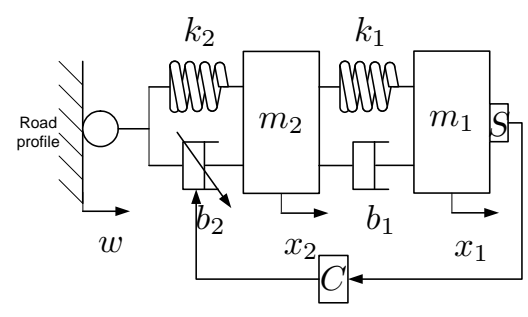

Fig. 1. A 2DOF quarter car model

dynamics is

$$
\begin{aligned}
& \dot{x}=A x+B_{1} w+B_{2}(x, \dot{w}) u, \\
& y=\ddot{x}_{2}=h(x, u, w, \dot{w}),
\end{aligned}
$$

where $x=\left(x_{1}, x_{2}, \dot{x}_{1}, \dot{x}_{2}\right)^{T}=\left(x_{1}, x_{2}, x_{3}, x_{4}\right)^{T}, u=b_{2}, w$ is the disturbance from the road profile, and

$$
\begin{gathered}
A=\left[\begin{array}{cccc}
0 & 0 & 1 & 0 \\
0 & 0 & 0 & 1 \\
-\frac{k_{1}}{m_{1}} & \frac{k_{1}}{m_{1}} & -\frac{b_{1}}{m_{1}} & \frac{b_{1}}{m_{1}} \\
\frac{k_{1}}{m_{2}} & -\frac{k_{1}+k_{2}}{m_{2}} & \frac{b_{1}}{m_{2}} & -\frac{b_{1}}{m_{2}}
\end{array}\right], \\
B_{1}=\left[\begin{array}{c}
0 \\
0 \\
0 \\
\frac{k_{2}}{m_{2}}
\end{array}\right], \quad B_{2}(x, \dot{w})=\left[\begin{array}{c}
0 \\
0 \\
0 \\
-\frac{\dot{x}_{2}-\dot{w}}{m_{2}}
\end{array}\right] .
\end{gathered}
$$

The system setup is non-unique. For instance, a semi-active actuator can be placed between $m_{1}$ and $m_{2}$. We however choose the aforementioned setup, because the dominant resonant mode of certain system is result from $m_{2}$, and an actuator between $m_{2}$ and the road profile is more effective in suppressing this resonance than the other setup. Placing a semi-active actuator between the road profile and $m_{2}$ however allows the effect of the disturbance derivative.

\section{B. Problem Statement}

Typically, the vibration reduction is formulated as a disturbance attenuation problem. This problem can be illustrated by Figure 2, where $G$ is the plant, and $z$ is the controlled variable, respectively. The vibration reduction problem is stated as follows.

Given the system (1) subject to the disturbance $w$ and other design constraints e.g. relative displacements, the power dissipative rate, and bounded control set, find a control $u$ which minimizes a cost function $J$.

If $w(t)$ is known, the treatment of optimal control can be followed to reject the vibration. Although it is impractical to assume the knowledge about the disturbance, the optimal control approach is, however, useful to derive the form of controller which optimally attenuates the disturbance, and to establish the bounds on the achievable performance of admissible controllers.

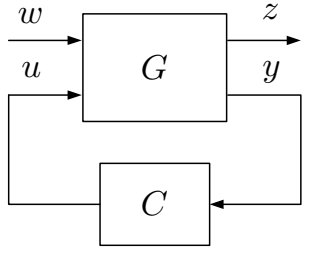

Fig. 2. Disturbance attenuation problem setup [16]

Generally, the cost $J$ reflects the ride comfort and physical constraints ensuring the safety operation. The ride comfort can be measured by norms of the first mass acceleration and its time derivative (jerk). In addition to the ride comfort, physical constraints are mainly the relative displacement between moving masses, the dissipative rate of power [12], and the bound of control. These constraints can be either included in $z$ or imposed as hard constraints.

This note only considers the ride comfort aspect, i.e., the performance criteria is $\mathcal{L}_{p}$-norm of the first mass acceleration. To design a control which minimizes $\left\|\ddot{x}_{1}(t)\right\|_{\infty}$ subject to $w(t)$ is an $\mathcal{L}_{\infty}$ control design problem, which is difficult to solve for nonlinear systems. We consider a cost function

$$
J=\left(\int_{0}^{T}\left|\ddot{x}_{1}(t)\right|^{p} \mathrm{~d} t\right)^{\frac{1}{p}} .
$$

When $p$ is sufficiently large, it is reasonable to assume that the corresponding control approximates the $\mathcal{L}_{\infty}$ control.

\section{Controller Design}

\section{A. Optimal Control}

We formulate the semi-active vibration reduction as a constrained optimal control problem with the cost function $J$ given by (2). The main purpose is to derive the structure of optimal control. We begin with defining the Hamiltonian

$H(x, \lambda, u, w, \dot{w})=\left|\ddot{x}_{1}(t)\right|^{p}+\lambda^{T}\left(A x+B_{1} w+B_{2}(x, \dot{w}) u\right)$.

Treating $w, \dot{w}$ as functions of time and applying the Minimum Principle, we have the necessary condition on the optimal control as follows

$$
H\left(x^{*}, \lambda^{*}, u^{*}, t\right)=\min _{u \in\left[b_{\min }, b_{\max }\right]} H\left(x^{*}, \lambda^{*}, u, t\right),
$$

where $x^{*}, u^{*}, \lambda^{*}$ are the optimal state, control, and costate trajectories, respectively. Since $\ddot{x}_{1}(t)$ is independent of $u$, the optimal control which minimizes $H\left(x^{*}, \lambda^{*}, u, t\right)$ is given by

$$
u^{*}= \begin{cases}b_{\max }, & \left(\lambda^{*}\right)^{T} B_{2}(x, \dot{w}) \leq 0, \\ b_{\min }, & \left(\lambda^{*}\right)^{T} B_{2}(x, \dot{w}) \geq 0,\end{cases}
$$

where $b_{\min }, b_{\max }$ are the upper and lower bounds of $u$.

Remark 3.1: The optimal control $u^{*}$ minimizing $\mathcal{L}_{p}$-norm of first mass acceleration necessarily takes the form of an onoff switch control. Given $\lambda^{*}=\left(\lambda_{1}^{*}, \ldots, \lambda_{4}^{*}\right)^{T}$, the optimal control can be simplified as

$$
u^{*}= \begin{cases}b_{\max }, & \lambda_{4}^{*}\left(x_{4}-\dot{w}\right) \geq 0, \\ b_{\min }, & \lambda_{4}^{*}\left(x_{4}-\dot{w}\right) \leq 0 .\end{cases}
$$


Various of existing semi-active control laws admit the form of (3). For instance, a conventional semi-active control law which implements the conceptual Ground-Hook (GH) strategy [5] is defined as follows

$$
u=\left\{\begin{array}{l}
\min \left\{\frac{b x_{4}}{x_{4}-\dot{w}}, b_{\max }\right\}, \quad x_{4}\left(x_{4}-\dot{w}\right) \geq 0, \\
b_{\min }, \quad x_{4}\left(x_{4}-\dot{w}\right)<0,
\end{array}\right.
$$

where $\lambda_{4}^{*}$ in (3) is replaced with $b x_{4}$, and $b$ is the damping coefficient of a damper attached between the second mass and the inertial reference. The amplitude of control $u$ (4) can be piecewise continuous.

Remark 3.2: The key to solve the optimal control $u^{*}$ is to solve for $\lambda_{4}^{*}$. Theoretically, one can obtain $u^{*}(t)$ by solving a boundary value problem. The resultant optimal control relies on the disturbance and its time derivative, and the initial state condition. Alternatively, one can follow the dynamic programming approach to solve $u^{*}(t)$ from the well-known Hamilton-Jacobi-Bellman (HJB) equation, which will be two switched nonlinear partial differential equations

$$
\begin{aligned}
& \frac{\partial V}{\partial x} f+\frac{\partial V}{\partial x} B_{2} b_{\text {min }}+q(x)+\frac{\partial V}{\partial t}=0, \text { if } \frac{\partial V}{\partial x} B_{2}<0 \\
& \frac{\partial V}{\partial x} f+\frac{\partial V}{\partial x} B_{2} b_{\text {max }}+q(x)+\frac{\partial V}{\partial t}=0, \text { if } \frac{\partial V}{\partial x} B_{2} \geq 0
\end{aligned}
$$

where $f=A x+B_{1} w, g=B_{2}, q(x)=\left|\ddot{x}_{1}(t)\right|^{p}$ is the Lagrangian. Given the value function $V(x, t)$ solved from (5) and denoting $\lambda^{*}=\partial V / \partial x$, the $u^{*}(t)$ is the same as (3). In general, solving the time varying HJB (5) is difficult.

Besides the difficulty to solve the closed-form optimal control law, another major weakness of the optimal control approach is that it requires the perfect knowledge about disturbance and its time derivative, and state. It is therefore not feasible to apply optimal control to the semi-active systems in practice. This motivates us to develop an implementable sub-optimal control.

\section{B. Sub-Optimal Control}

We propose a controller which takes the exact same form as the optimal control (3). The idea of selecting this control structure is to mimic the optimal control by approximating the optimal costate trajectory $\lambda_{4}^{*}$. The sub-optimal control law is defined as follows

$$
u= \begin{cases}b_{\max }, & \varphi(\hat{x}, y)\left(x_{4}-\dot{w}\right)>0, \\ b_{\min }, & \varphi(\hat{x}, y)\left(x_{4}-\dot{w}\right) \leq 0,\end{cases}
$$

where $\varphi$ is a function of the measurement $y$ and the estimate of the original system state. $\varphi$ is used to approximate $\lambda_{4}^{*}$. Note that a number of existing semi-active controls are special cases of the control (6). For instance, with $\varphi=$ $\hat{x}_{4}$, (6) reduces to Acceleration Driven Damping (ADD) as follows [17]

$$
u= \begin{cases}b_{\max }, & \dot{x}_{4}\left(x_{4}-\dot{w}\right) \geq 0, \\ b_{\min }, & \dot{x}_{4}\left(x_{4}-\dot{w}\right)<0 .\end{cases}
$$

When $\varphi$ is a linear combination of state, (6) reduces to the clipped optimal control [18].
A natural choice of $\varphi$ is to mix the well-known GH strategy (4) and ADD strategy (7). This is because the frequency characteristics of the closed-loop systems using these two controls are complementary [19], i.e., switch control based on the $\mathrm{GH}$ achieves good performance in isolating the low frequency disturbances, and ADD is good at attenuating the high frequency disturbance. On the other hand, given $p=2$, and matching the order of $x$ in (5), we know that $V$ is a quadratic function of $x$, which implies $\lambda_{4}$ is a linear function of state. Given the second mass acceleration, we propose the sub-optimal control law as follows

$$
u= \begin{cases}b_{\max }, & \left(c_{1} \hat{\ddot{x}}_{1}+c_{2} \dot{x}_{4}+\hat{x}_{4}\right)\left(\hat{x}_{4}-\hat{\dot{w}}\right) \geq 0, \\ b_{\text {min }}, & \text { otherwise }\end{cases}
$$

where $c_{1}, c_{2}$ are constants, $\hat{\ddot{x}}_{1}$ is the estimated acceleration of the first mass, $\hat{x}_{4}$ is the estimated velocity of the second mass, and $\hat{x}_{4}-\hat{\dot{w}}$ is the estimated relative velocity of the semi-active actuator.

Remark 3.3: Allowing $\varphi$ be a function of the second mass acceleration, estimates of the first mass acceleration and the second mass velocity is because as shown later, all these signals are relatively easy to obtain. Assuming constant parameters $c_{1}, c_{2}$ is to simplify the performance analysis of the resultant closed-loop system and the tuning of controller. This is however not necessary and can be generalized. Allowing a more general $\varphi$ potentially increases the achievable performance of vibration reduction at the expense of a complex tuning.

\section{Stability Analysis}

We aim to establish that the closed-loop semi-active system (1) with control (8) is Input-to-State Stable (ISS), where the disturbance is treated as input.

Proposition 3.4: System (1) with the control (8) is ISS.

Proof: The closed-loop semi-active system can be formulated as the following switched system

$$
\begin{array}{ll}
\dot{x}=A_{1} x+\psi_{1}(w, \dot{w}), & u=b_{\max }, \\
\dot{x}=A_{2} x+\psi_{2}(w, \dot{w}), & u=b_{\min } .
\end{array}
$$

We first study the stability of the homogenous part of (9)

$$
\begin{array}{lll}
\Sigma_{1}: & \dot{x}=A_{1} x, & u=b_{\max }, \\
\Sigma_{2}: & \dot{x}=A_{2} x, & u=b_{\min } .
\end{array}
$$

We assume system (10) switches arbitrarily, which is more general than the closed-loop semi-active case where control (8) switches by state. Taking the Lyapunov candidate as the physical energy of the unforced switch system (10), we have $\dot{V}<0, \forall x \neq 0$ because of the existence of dampers in the physical system. Denoting $V=x^{T} P x, P>0$, and $\dot{V} \leq$ $x^{T} Q x$, we know $Q$ is negative definite.

To show that (9) is ISS, we use the same $V$. Its time derivative is

$$
\begin{aligned}
\dot{V} & \leq x^{T} Q x+2\|P x\| \max \left\{\left\|\psi_{1}\right\|_{\infty},\left\|\psi_{2}\right\|_{\infty}\right\} \\
& \leq x^{T} Q x+\epsilon x^{T} P^{2} x+\frac{1}{\epsilon}\left\|\psi_{3}\right\|_{\infty},
\end{aligned}
$$


where $\epsilon>0$, and $\psi_{3}$ satisfies

$$
\left\|\psi_{3}\right\|_{\infty} \geq \max \left\{\left\|\psi_{1}\right\|_{\infty}^{2},\left\|\psi_{2}\right\|_{\infty}^{2}\right\} .
$$

One can always take a sufficiently small $\epsilon$ s.t.

$$
x^{T}\left(Q+\epsilon P^{2}\right) x \leq-\mu\|x\|_{2}^{2} .
$$

Hence, we have

$$
\dot{V} \leq-\mu\|x\|_{2}^{2}+\frac{1}{\epsilon}\left\|\psi_{3}\right\|_{\infty},
$$

and

$$
\dot{V} \leq-(1-\theta)\|x\|_{2}^{2}, \quad \forall\|x\| \geq \sqrt{\frac{\left\|\psi_{3}\right\|_{\infty}}{\theta \epsilon}},
$$

where $0<\theta<1$. Applying [20, Thm. 4.19], we conclude that system (9) is ISS w.r.t. $w, \dot{w}$.

Remark 3.5: System (9) is ISS implies that it is Bounded Input Bounded Output stable. One can alternatively show that system (10) is globally exponentially stable by solving the following Linear Matrix Inequalities (LMIs)

$$
\begin{aligned}
& A_{1}^{T} P+P A_{1} \leq 0, \\
& A_{2}^{T} P+P A_{2} \leq 0,
\end{aligned}
$$

whose solution is guaranteed by the system's dissipativity. The $\mathcal{L}_{2}$ stability of the semi-active system can be similarly analyzed and posed as the solvability of a set of LMIs. Slightly different from the ISS case, we need to consider an augmented semi-active system which includes the disturbance model.

\section{Implementation of Control (8)}

The state feedback control (8) requires the knowledge of the absolute velocity of the second mass $x_{4}$, and the relative velocity between the second mass and road profile, $x_{4}-$ $\dot{w}$. Both of states are difficult or expensive to measure in practice. The approximation of the second mass velocity $x_{4}$ has been discussed and obtained by passing the second mass acceleration through a band-pass filter [21]. We focus on the estimation of the acceleration of the first mass $\ddot{x}_{1}$ and the relative velocity $x_{4}-\dot{w}$. The estimation of the sign of the relative velocity is critical here because it determines the time to turn on and off the actuator.

For the relative velocity $x_{4}-\dot{w}$, we notice the dynamics of $x_{4}$ can be rewritten as

$$
\dot{x}_{4}=-\frac{m_{1}}{m_{2}} \ddot{x}_{1}-\frac{k_{2}}{m_{2}}\left(x_{2}-w\right)-\frac{u(t)}{m_{2}}\left(\dot{x}_{2}-\dot{w}\right) .
$$

Assuming the knowledge of the first mass and second mass accelerations $\ddot{x}_{1}, \dot{x}_{4}$, we write the dynamics of $\eta=x_{2}-w$

$$
\dot{\eta}=\frac{-1}{u(t)}\left(k_{2} \eta+m_{2} \dot{x}_{4}+m_{1} \ddot{x}_{1}\right) \text {. }
$$

Introducing a linear time-varying filter

$$
\dot{\hat{\eta}}=\frac{-1}{u(t)}\left(k_{2} \hat{\eta}+m_{2} \dot{x}_{4}+m_{1} \ddot{x}_{1}\right),
$$

we have the estimation of the relative velocity, which converges to the true value of $\eta$ exponentially. This is obvious by verifying that the zero solution of the resulting error dynamics $\tilde{\eta}=\eta-\hat{\eta}$ is exponentially stable,

$$
\dot{\tilde{\eta}}=-\frac{k_{2}}{u(t)} \tilde{\eta}
$$

To estimate the acceleration of the first mass, we examine the dynamics of $\left(x_{1}, x_{3}\right)$ by treating $\left(x_{2}, x_{4}\right)$ as output, and have the transfer function from the second mass displacement to the first mass displacement

$$
G_{2}=\frac{X_{1}(s)}{X_{2}(s)}=\frac{b_{1} s+k_{1}}{m_{1} s^{2}+b_{1} s+k_{1}} .
$$

Since the transfer function from the second mass acceleration to the first mass acceleration is $G_{2}$ as well, we introduce a linear time invariant filter as follows

$$
\begin{aligned}
\dot{\hat{x}}_{1} & =\hat{x}_{3}, \\
\dot{\hat{x}}_{3} & =-\frac{k_{1}}{m_{1}} \hat{x}_{1}-\frac{b_{1}}{m_{1}} \hat{x}_{3}+\frac{1}{m_{1}} \dot{x}_{4}, \\
\hat{y} & =k_{1} \hat{x}_{1}+b_{1} \hat{x}_{3} .
\end{aligned}
$$

The output of (13) is the estimation of the first mass acceleration which converges to the the first mass acceleration exponentially. We finally implement the output feedback sub-optimal control as (8), (12), and (13), where $\ddot{x}_{1}$ in (12) is replaced with $\hat{\ddot{x}}_{1}$. The implementation of control merely measures the second mass acceleration, thus reduce the hardware cost of the entire system.

Remark 3.6: The globally exponential stability of the dynamics of estimation error is established because the dynamics of $x_{1}$ and $x_{4}-\dot{w}$ are stable. The proposed estimator given by (12) and (13) does not have freedom in controlling the convergence speed of the resultant error dynamics.

\section{PERformanCE ANALYSis}

Given the sub-optimal control (8), we would like to tune the controller, and evaluate the performance of the resultant closed-loop system. The tuning and evaluation is based on two criteria: the peak to peak acceleration of the first mass, and $\mathcal{L}_{2}$-norm of the acceleration of the first mass. Assuming the first mass acceleration $z(t), t \in[0, T]$, the peak to peak acceleration is defined as

$$
\mathrm{P} 2 \mathbf{P}=\max _{t \in[0, T]} z(t)-\min _{t \in[0, T]} z(t) .
$$

The performance metric $\mathrm{P} 2 \mathrm{P}$ is closely relate to the $\infty$ norm. However, they are different and the $\infty$-norm is more conservative than $\mathrm{P} 2 \mathrm{P}$. This is evident by noticing

$$
\mathrm{P} 2 \mathrm{P} \leq 2\|z\|_{\infty} .
$$

\section{A. $\mathcal{L}_{2}$ Analysis}

This subsection is to tune the control (8) so that it minimizes the $\mathcal{L}_{2}$-norm cost functional. This approach is based on the assumption that the closed-loop semi-active system can be approximated fairly well by a linear system. Simulation of the semi-active system reveals the dominance of the superposition principle. That is, $\Psi(a w(t))=a \Psi(w(t))$, where $a$ is constant, and $\Psi: \mathcal{L}_{2} \rightarrow \mathcal{L}_{2}$ is the nonlinear operator 
characterizing the input output model of the semi-active system. On the other hand, we find $\Psi\left(w_{1}(t)+w_{1}(t)\right) \neq$ $\Psi\left(w_{1}(t)\right)+\Psi\left(w_{2}(t)\right)$, if $w_{1}(t)$ and $w_{2}(t)$ are different.

Figure 3 illustrates how we obtain the approximate $\mathcal{L}_{2}$ gain of the semi-active system. A single frequency sinusoid signal $r$ is used to excite the closed-loop semi-active system, then a frequency dependent $\mathcal{L}_{2}$ gain is computed as follows

$$
\gamma(\omega)=\sqrt{\frac{\int_{0}^{T} z^{2}(t) \mathrm{d} t}{\int_{0}^{T} r^{2}(t) \mathrm{d} t}} .
$$

We also employ the $W_{1}(s)$ to incorporate the low-pass model of the disturbance which is obtained by estimating the power spectrum of the real disturbance data. The cost function is defined as

$$
J_{1}\left(c_{1}, c_{2}\right)=\int_{\omega_{2}}^{\omega_{1}} \gamma^{2}(\omega) \mathrm{d} \omega .
$$

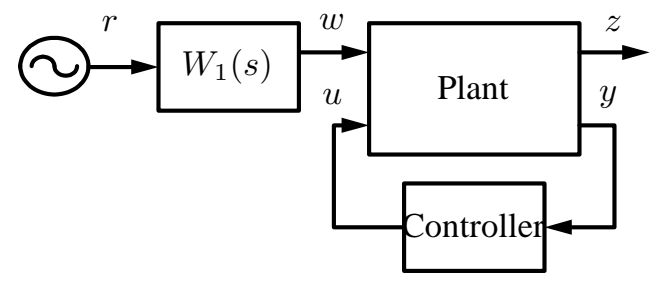

Fig. 3. Schematics of $L_{2}$ analysis

It is clear that $J_{1}$ is a function of $\zeta, \omega_{n}, \omega_{1}, \omega_{2}, b_{\min }, b_{\max }$ as well as $c_{1}, c_{2}$. For simplicity, we take

$$
\begin{aligned}
r & =1 e-3 \sin (\omega t), \\
\zeta & =0.707, \quad \omega_{n}=12 \mathrm{rad} / \mathrm{sec}, \\
\omega_{1} & =1 \mathrm{rad} / \mathrm{sec}, \quad \omega_{2}=35 \mathrm{rad} / \mathrm{sec} .
\end{aligned}
$$

Numerical simulation is used to investigate how the parameters $c_{1}, c_{2}$ affect the performance cost. Introducing the sets of $U_{1}, U_{2}$ which are the domain of $c_{1}, c_{2}$ respectively,

$$
\begin{aligned}
& U_{1}=\{0,0.05,0.10,0.15,0.20,0.25\}, \\
& U_{2}=\{0, \pm 0.05, \pm 0.10, \pm 0.15, \pm 0.20, \pm 0.25\} .
\end{aligned}
$$

We simulate the semi-active system by enumerating $c_{1}, c_{2}$ over $U_{1}, U_{2}$, and have the simulation result as shown in Figure 4. Hence, the sub-optimal control parameters, which minimize $J_{1}$ are $S_{1}=\left\{c_{1}=0.05, c_{2}=0\right\}$.

Remark 4.1: Here we perform exhaustive search for control parameters. Various approaches including gradient-based adaptation, extremum-seeking can also be applied to tune the controller.

\section{B. P2P Tuning}

Another important criteria of ride comfort is the peak acceleration of the first mass. Control minimizing this cost can be approximated by a $\mathcal{L}_{\infty}$ control which minimizes the $\infty$-norm of the controlled variable. The control design of an LTI plant to reject a persistent disturbance subject to $|z(t)|_{\infty}$ has been formulated as a $\mathcal{L}_{1}$ control and investigated in [22], [23]. The resulting controller is difficult to implement. Other

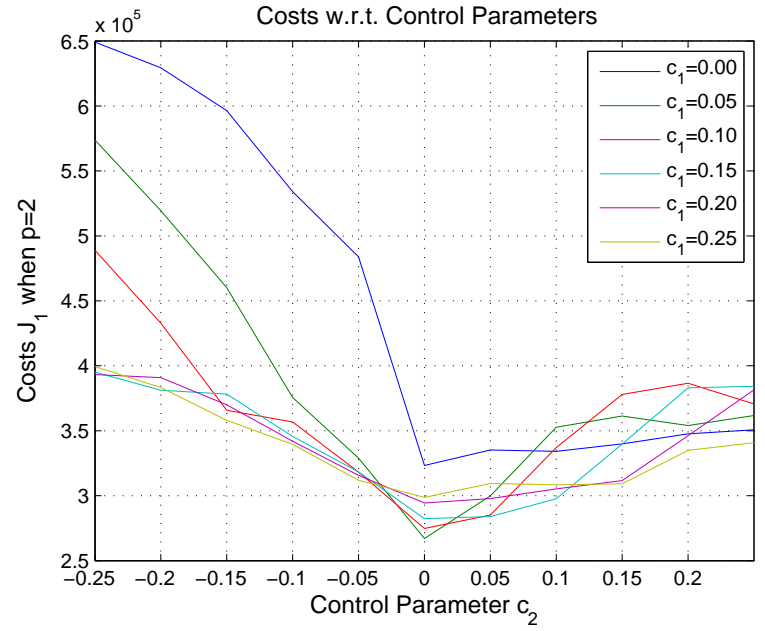

Fig. 4. Costs of the Semi-Active System w.r.t. $c_{1}, c_{2}$

work [24] attempts to solve the bounded output peak problem using LMIs, which might be conservative. On the other hand, the $\mathcal{L}_{1}$ control design for nonlinear system remains open. Hence, numerical simulation of nonlinear system subject to certain signals will be performed to tune the control parameters which minimizes the $\mathrm{P} 2 \mathrm{P}$ cost.

We evaluate the P2P of the semi-active system subject to triangular signals with different frequencies, which partially capture characteristics of the level variation of the road profile. We define the cost function

$$
J_{2}\left(c_{1}, c_{2}\right)=\int_{\omega_{1}}^{\omega_{2}} \frac{\mathrm{P}_{2} \mathrm{P}_{p}(\omega)-\mathrm{P}_{2} \mathrm{P}_{s a}(\omega)}{\mathrm{P}_{2} \mathrm{P}_{p}(\omega)} \mathrm{d} \omega,
$$

where $\mathrm{P} 2 \mathrm{P}_{p}(\omega)$ and $\mathrm{P} 2 \mathrm{P}_{s a}(\omega)$ are $\mathrm{P} 2 \mathrm{P}$ for the passive and semi-active systems respectively. The cost $J_{2}$ means the improvement of vibration reduction of the semi-active system over the passive system. Different from the cost $J_{1}$ which is to be minimized, the cost $J_{2}$ is to be maximized.

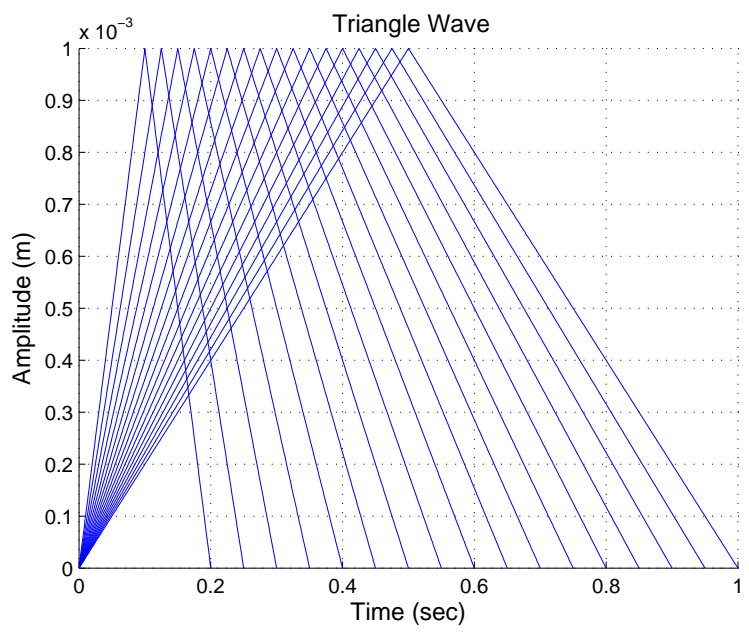

Fig. 5. Triangular Inputs with Different Frequencies

To evaluate the cost $J_{2}$ for every set of parameters over $U_{1}, U_{2}$, a number of triangular waves are treated as $w$ in 
Figure 5 and are fed into the semi-active system. Given a set of parameters $c_{1}, c_{2}$, the cost functional $J_{2}$ is computed according to (15) but using summation over a discrete frequency points instead of integral over a frequency interval. We finally have the performance curves shown in Figure 6. Maximizing $J_{2}$ over $U_{1}, U_{2}$, shown in Figure 6, gives control parameters $S_{2}=\left\{c_{1}=0.25, c_{2}=-0.05\right\}$.

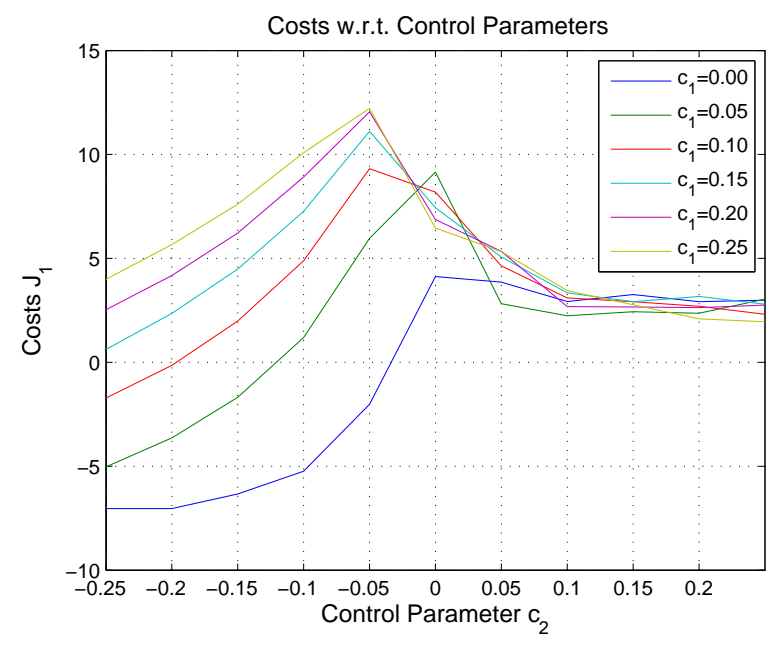

Fig. 6. Costs of the Semi-Active System w.r.t. $c_{1}, c_{2}$

\section{Validation}

We simulate a passive system, which is the case when $u=b_{\min }$, and four semi-active control laws: the proposed sub-optimal control (8) with parameters given by $S_{1}$ and $S_{2}$, conventional controls (4) and (7), respectively. The real level variation data of the road profile is treated as the disturbance. The simulation results are summarized in Table I. We can see the sub-optimal control (8) with $S_{1}$ minimizing $\mathcal{L}_{2}$ gain has the lowest level of 2-norm cost, and (8) with $S_{2}$ minimizing $\mathrm{P} 2 \mathrm{P}$ shows the best performance in reducing the P2P. This demonstrates the effectiveness of the proposed control and the tuning methodology.

TABLE I

Simulation Results of Semi-Active Controls

\begin{tabular}{c|c|c|c|c|c}
\hline Costs & Passive & $(8)$ with $S_{1}$ & $(8)$ with $S_{2}$ & $(4)$ & $(7)$ \\
\hline$\|z\|_{2}$ & 6.6092 & 3.4117 & 3.5920 & 3.5106 & 4.3849 \\
\hline P2P & 0.2495 & 0.2199 & 0.1815 & 0.2451 & 0.2386 \\
\hline
\end{tabular}

\section{CONCLUSION}

This paper considers the control design for vibration reduction of transportation systems using semi-active actuators. The form of the optimal disturbance attenuation of the semiactive system is discussed and shown to be an on-off switch strategy. A sub-optimal control is proposed and implemented using one sensor measurement. Performance analysis of the sub-optimal control subject to cost functionals is investigated using numerical computation. The semi-active system with the sub-optimal control is simulated to demonstrate its effectiveness.

\section{REFERENCES}

[1] D. C. Karnopp, "Theoretical limitations in active vehicle suspensions," Vehicle Syst. Dyn., vol. 15, no. 1, pp. 41-54, 1986.

[2] D. C. Karnopp, M. J. Crosby, and R. A. Harwood, "Vibration control using semi-active force suspension," Trans. ASME, J. Eng. Ind., vol. 96, no. 2, pp. 619-626, 1974.

[3] E. J. Krasnicki, "Comparison of analytical and experimental results for a semi-active vibration isolator," in Shock and Vibration Bulletin, vol. 50. Washington, DC: The Shock and Vibration Information Center, Naval Research Laboratory, 1980.

[4] S. J. Dyke, B. F. Spencer Jr., M. K. Sain, and J. D. Carlson, "Modeling and control of magnetorheological dampers for seismic response reduction," Smart Mater. Struct., vol. 5, no. 5, pp. 565-575, Oct. 1996.

[5] M. Valásek and M. Novák, "A new concept of semi-active control of truck's suspension," in Proceedings of International Symposium on Advanced Vehicle Control, Aachen, Germany, Jun. 1996, pp. 141-151.

[6] D. Hrovat, "Applications of optimal control to advanced automotive suspension design," Trans. ASME, J. Dyn. Sys. Meas. Control, vol. 115, no. 2B, pp. 328-342, Jun. 1993.

[7] H. Du, S. K. Yim, and J. Lam, "Semi-active $\mathrm{H}_{\infty}$ control of vehicle suspension with magneto-rheological dampers," J. Sound Vib., vol. 283, no. 3-5, pp. 981-996, May 2005.

[8] D. Hrovat, D. L. Margolis, and M. Hubbard, "An approach toward the optimal semi-active suspension," Trans. ASME, J. Dyn. Sys. Meas. Control, vol. 111, no. 3, pp. 288-296, 1988.

[9] H. E. Tseng and J. K. Hedrick, "Semi-active control laws-optimal and sub-optimal," Vehicle Syst. Dyn., vol. 23, no. 1, pp. 545-569, 1994.

[10] G. Leitmann, "Semiactive control for vibration attenuation," J. Intel. Mat. Syst. Str., vol. 5, no. 6, pp. 841-846, 1994.

[11] N. H. McClamroch and H. P. Gavin, "Closed loop structure control using electrorheological dampers," in Proc. 1995 ACC, vol. 6, Evanston, IL, Jun. 1995, pp. 4173-4177.

[12] N. Giorgetti, A. Bemporad, H. E. Tseng, and D. Hrovat, "Hybrid model predictive control application towards optimal semi-active suspension," Int. J. Control, vol. 79, no. 5, pp. 521-533, May. 2006.

[13] S. Ohsaku, T. Nakayama, I. Kamimura, and Y. Motozono, "Nonlinear $\mathrm{H}_{\infty}$ control for semi-active suspension," JSAE Review, vol. 20, no. 4, pp. 447-452, 1999.

[14] E. Shimizu, M. Sampei, and M. Koga, "Design of a nonliear $\mathrm{H}_{\infty}$ state feedback controller for bilinear systems with nonlinear weight," in Proc. 36th CDC, San Diego, CA, Dec. 1997, pp. 2323-2324.

[15] C. Poussot-Vassala, C. Speltab, O. Senamec, S. Savaresid, and L. Dugardc, "Survey and performance evaluation on some automotive semi-active suspension control methods: A comparative study on a single-corner model," Annual Reviews in Control, vol. 36, pp. 148$160,2012$.

[16] T. Chen and B. A. Francis, Optimal Sampled-data Control systems. New York: Springer-Verlag, 1995.

[17] S. M. Savaresi, E. Silani, and S. Bittanti, "Accelertaion-driven-damper (ADD): An optimal contorl algorithm for comfort-oriented semi-active suspensions," Trans. ASME, J. Dyn. Sys. Meas. Control, vol. 127, no. 2, pp. $218-229,2005$.

[18] S. J. Dyke and B. F. Spencer Jr., "A comparison of semi-active control strategies for the MR damper," in Proceedings of International Conference on Intelligent Information Systems, Bahamas, Grand Bahama Island, 1997, pp. 580-584.

[19] S. M. Savaresi and C. Spelta, "Mixed Sky-Hook and ADD: approaching the filtering limits of a semi-active suspension," Trans. ASME, J. Dyn. Sys. Meas. Control, vol. 129, no. 4, pp. 382-392, Jul. 2007.

[20] H. K. Khalil, Nonlinear Systems, 3rd ed. Englewood Cliffs, NJ: Prentice-Hall, 2002.

[21] D. Margolis, "The response of active and semi-active suspensions to realistic feedback signals," Vehicle Syst. Dyn., vol. 11, no. 5, pp. 267282, 1982.

[22] M. A. Dahleh and J. B. Pearson, " $l^{1}$-optimal compensators for continuous-time systems," IEEE Trans. Automat. Contr, vol. AC-32, no. 10 , pp. 889-895, Oct. 1987.

[23] — , " $l^{1}$-optimal feedback controllers for MIMO discrete-time systems," IEEE Trans. Automat. Contr., vol. AC-32, no. 4, pp. 314-322, Apr. 1987.

[24] S. Boyd, L. E. Ghaoui, E. Feron, and V. Balakrishnan, Linear Matrix Inequalities in System and Control Theory. Society for Industrial and Applied Mathematics (SIAM), 1994. 